\title{
Path Loss Modeling for UWB Creeping Waves Around Human Body
}

\author{
Timo Kumpuniemi, Matti Hämäläinen, Juha-Pekka Mäkelä, Jari Iinatti \\ Centre for Wireless Communications \\ University of Oulu \\ Oulu, Finland \\ forename.surname@oulu.fi
}

\begin{abstract}
This article presents path loss models for creeping waves propagation around a human body at ultra-wideband frequency of 2-8 GHz. The results are based on measurements in an anechoic chamber using a vector network analyzer. Three antenna types are used: prototype dipole and double loop antennas together with the commercial Skycross antenna. The antennas are attached horizontally at the chest level and the waist level at in total 12 locations. Path loss values are defined from the first arriving paths of the channel impulse responses. Path loss models are developed for upper and lower horizontal body levels separately and for all available channels when also the cross channels between the levels are considered. The path loss exponents vary between $\mathbf{9 . 2 - 1 2 . 7}$ being clearly a higher result than previously reported. The double loop antenna has the lowest exponents and the commercial antenna the highest ones. No clear difference can be noted between the body and waist levels or when observing all channels. The scattering term of the path loss can be modeled with the generalized extreme value and generalized Pareto distributions.
\end{abstract}

Keywords—propagation; surface waves; ultra-wideband.

\section{INTRODUCTION}

The development of electronics and its manufacturing technologies together with the advances in wireless communications and computing efficiency have enabled the miniaturization trend of electronic devices. Today their size is so small that they can be located in totally new environments and be integrated in many appliances. One quite popular environment is the immediate vicinity of a human body creating a concept called a body area network (BAN) or wireless BAN (WBAN). Products for this context exist in the area of sports and well-being where users can measure body parameters with devices attached to their bodies in order to verify that exercises have been optimally effective and to follow the progress of their physical condition. They may monitor the level of their daily activity or the quality of sleep and save the data to create their personal well-being history.

In the medical applications, the small wireless devices with sensors attached on the body can provide a large number of benefits as the lack of wires advances the mobility of the patients and enables remote monitoring of patients in their homes instead in medical institutions. In addition to the increased quality of life of the patients and the working conditions of the medical staff, the need for resources in hospital and medical wards is eased up decreasing the costs of the society. Also the quality of medical care is increased as the small and user-friendly devices can collect data more frequently instead of seldom taken samples of a body parameter in a laboratory of a hospital or primary care ward.

The link distances between devices in a WBAN are short. One very suitable technology in this case is the ultra-wideband (UWB) technique providing low transmission power, good positioning, ranging and tracking characteristics. A technical structure can be simple leading to a small size and low unit cost $[1,2]$. The transmission is noise-like, not interfering communications of other wireless systems. An UWB system also tolerates interference from other systems. IEEE published the standard IEEE802.15.6 in 2012 where the impulse radio UWB was chosen to be the mandatory physical layer technique [3]. It contains also specifications for the frequency modulation UWB together with several narrowband waveforms.

The scope of this paper is to examine the UWB signal propagation around a human torso. In this case, the signal propagates mainly as a creeping wave, [4], following the body surface and its shape beyond line-of-sight conditions. In [5], the UWB signal propagation at the human head is reported at a 1.5-8 GHz bandwidth. Analytical model and measurements are presented in [6] for a narrowband signal at $2.45 \mathrm{GHz}$ around the head and waist. In [7], measurements around a human body at 3.1-10.6 GHz bandwidth are published. In [8], a simulation based UWB channel model around a human body is available and [9] extends the work by reporting the measurement results. Narrowband results at $900 \mathrm{MHz}$ and $2.45 \mathrm{GHz}$ around a human body can be found from [10].

The work in this paper is based on measurements carried out at the 2-8 GHz frequency band in an anechoic chamber by using a vector network analyzer (VNA). The main focus is to firstly extend the results in [11] by examining the available path loss (PL) in difficult channel conditions when the reflections from the structures near the body are excluded. Secondly, three antenna types are used to examine their differences: the prototype dipole and double loop antennas and the commercial Skycross antenna. The same commercial antenna is applied in $[5,7,9]$. The prototype antennas are used, e.g., in [11-16] in different WBAN measurement scenarios.

\section{EXPERIMENT SETUP}

The measurements were performed in an anechoic chamber with a floor size of $2.45 \mathrm{~m}$ by $2.45 \mathrm{~m}$. It was built with 
movable absorber blocks located in an electromagnetic compatibility room. A $181 \mathrm{~cm}$ tall male at his early twenties with a weight of $76 \mathrm{~kg}$ was serving as a test person. He was wearing a normal cotton T-shirt and jeans. All metal containing items as the belt, shoes, watch etc. were absent.

A four-port VNA (Rohde \& Schwarz ZVA8) located outside the anechoic chamber was set to measure the $2-8 \mathrm{GHz}$ frequency range 100 times in each measurement collecting 1601 points across the bandwidth. One recording lasted 90 seconds. All scattering parameters were recorded. The intermediate bandwidth of the VNA was $100 \mathrm{kHz}$, sweep time $288.18 \mathrm{~ms}$ and the transmit power $+10 \mathrm{dBm}$. Four eight meters long measurement cables (Huber \& Suhner SUCOFLEX 104PEA) were applied to connect the VNA to the antennas.

The research was repeated with three different antenna types: prototype planar dipole and double loop antennas have reported in detail in [12-14] and the commercial Skycross SMT-3TO10M-A antenna. The antennas are shown in Fig. 1 where the ruler is in centimeters.

Even though the prototype antennas do not contain an embedded balun structure, no external baluns were used. The Skycross antenna does not need a balun due to its unbalanced structure. It was observed that no notable difference exists between the antenna operations due to the lack of baluns. Furthermore, external applicable baluns are difficult -perhaps even impossible- to find at the frequency ranges of our work.

\section{EXPERIMENT SCENARIO}

Twelve measurement locations were selected as depicted in Fig. 2. The antennas spots are selected at two horizontal planes with a distance of approx. $25 \mathrm{~cm}$ from each other. The horizontal distance between the neighbouring antennas in a plane is approx. $5 \mathrm{~cm}$ considering the practical issues due to antennas, cables and body shapes. The two antennas at spots A and $\mathrm{B}$ are kept constant during all measurements. The other two antennas are installed at spots $1-10$ in pairs so that locations 1-6, 2-7 etc. form pairs. In practice, five combinations of four antennas are measured as listed in Table I. Since both forward and reverse channels are measured, one plane results ten channels (1000 separate frequency sweeps) for the data analysis. When also all cross-channels between the upper and lower horizontal plane are considered, the number of channels is 60, i.e. 6000 separate frequency sweeps.

The antennas are attached on the body with elastic bands and paper tape. To keep the antenna-body distance constant, a

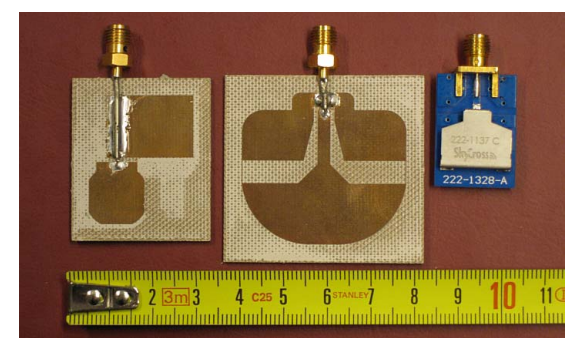

Fig. 1. The prototype dipole (left), the prototype double loop (center) and Skycross (right) antenna.
$20 \mathrm{~mm}$ thick piece of ROHACELL $31 \mathrm{HF}$ is inserted between an antenna and the body. In $[12,13]$ this distance was found to be a good choice with respect to both the antenna matching and the path gain as it has minimal effect on both parameters compared to free space.

During the measurements, the test subject was standing still. He kept his arms up as we wanted to focus only on the propagation around the torso. With the hands down on the sides of the torso the PL between some of the links from front to backside of the body would be very difficult to detect due to the high signal attenuation.

PL models are developed in three cases for all antennas. The upper and lower planes in Fig. 2 are examined separately and thirdly all channels including the cross channels available according to Table I are included.

\section{DATA ANALYSIS AND RESUlts}

\section{A. Data Post-Processing}

The inverse fast Fourier algorithm (IFFT) was applied to the frequency domain measurement data to extract the corresponding channel impulse responses (CIRs) in time domain. Since the signal bandwidth is $6 \mathrm{GHz}$, the obtained time domain resolution is $1 / 6 \mathrm{GHz} \approx 0.167 \mathrm{~ns}$. Assuming the velocity of light as the propagation speed, it corresponds to a resolution of $5 \mathrm{~cm}$ in distance. The CIR approach has the advantage that the signal components propagating around the body as creeping waves can be separated from the components originating from the reflections close to the human body. Even though the measurements took place in an anechoic chamber, we noticed that the absorber blocks produce weak reflections that in the extreme cases are far stronger than the diffracted components around the torso. These components can be removed from the CIRs to avoid their possible skewing effect on the results. As an example, Fig. 3 presents CIRs for the links A-1 and A-5 with the Skycross antenna. As noted, in the link A-5 the absorber reflections are much stronger than the creeping wave part propagated around the body. An analysis in

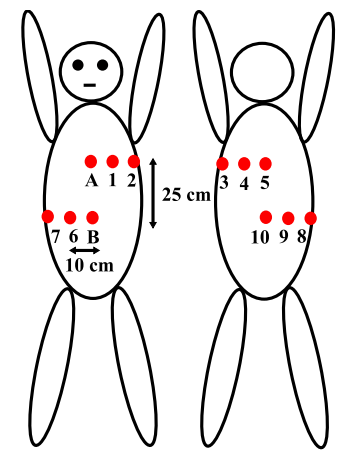

a)

b)

Fig. 2. On-body antenna locations a) front b) back.

TABLE I. MEASUREMENT LOCATION COMBINATIONS

\begin{tabular}{|ll|l|}
\hline $\mathrm{A}-\mathrm{B}-1-6$ & $\mathrm{~A}-\mathrm{B}-2-7$ \\
\hline $\mathrm{A}-\mathrm{B}-3-8$ & $\mathrm{~A}-\mathrm{B}-4-9$ \\
\hline \multicolumn{3}{|c|}{$\mathrm{A}-\mathrm{B}-5-10$} \\
\hline
\end{tabular}


the frequency domain would lead to an incorrect result when observing the creeping wave phenomenon since the reflected part would be present in the results.

During the IFFT no windowing was used. The windowing would reduce the sidelobe level in the response but the time domain resolution would be poorer. Furthermore, applying the windowing will cut off parts of the frequency domain signal causing loss in power level compared to the original signal. For accurate results, the power loss should be compensated.

The analysis is based on the first arriving paths (FAPs) of the CIRs. The corresponding link distance is calculated based on the FAP arrival time. The 100 sweeps per link are averaged into one CIR The FAPs are solved by finding the first CIR peak above the mean value of the 16 first CIR samples. Some links are so poor that the signals are almost buried in noise. The algorithm was unable to solve reliably the FAPs in these cases. Thus, the PLs above $110 \mathrm{~dB}$ are removed from the analysis.

In [11], it was noted that the dimensions of an antenna structures and their substrate both give rise to a delay in the signal propagation. As a result, the link distances appear to be longer than in reality when observed from a CIR. The error is different between different antennas and depends also on the relative position between the antennas. In short distances, as in body area networks, this error may have significant effect on the results. In [11] the average distance error was noted to be $90 \mathrm{~mm}$ and $109 \mathrm{~mm}$ for the prototype dipole and double loop, respectively. Measured in a similar way as in [11], the error is found to be $55 \mathrm{~mm}$ for the Skycross antenna. These errors are compensated in the analysis and the results in this paper. A more detailed information on the distance error due to the propagation delay in antennas is available in [11].

\section{B. Path Loss Model}

The PL model as a function of distance $d$ can be expressed as [17]

$$
P L(d)=P L\left(d_{0}\right)+10 n\left(\frac{d}{d_{0}}\right)+S[\mathrm{~dB}]
$$

where $P L$ is the PL in $\mathrm{dB}, P L\left(d_{0}\right)$ is the PL at the reference distance $d_{0}, n$ is the PL exponent and $S$ is a random scattering term with the standard deviation of $\sigma$. The value for $d_{0}$ is selected to be $50 \mathrm{~mm}$ to guarantee that $d \geq d_{0}$.

Fig. 4 shows graphically the empirical PLs as dots in the case when all channels are analyzed. The solid line is the fitting line obtained by using the first order linear least squares polynomial fitting method. Visually observed the Skycross antenna has the steepest slope in the fitted line and the double loop has the gentlest one. Generally speaking the PLs grow rapidly for all antennas. Fig. 5 presents the case for all antennas when the focus is on the upper horizontal plane. The fitted lines behave quite similarly as in Fig. 4.

The numerical values are gathered in Table II. The texts "Upper" and "Lower" denote that the focus is on upper and lower plane shown in Fig. 1. "All" represents the case when all available channels are included. The PL exponents vary
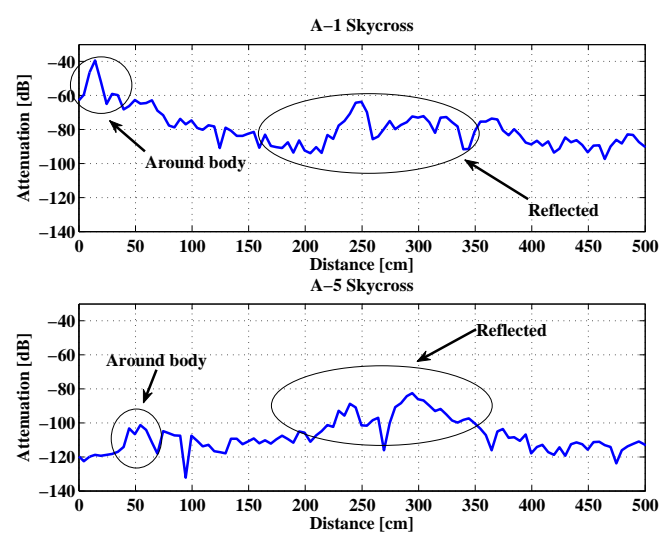

Fig. 3. Example CIRs for Skycross.
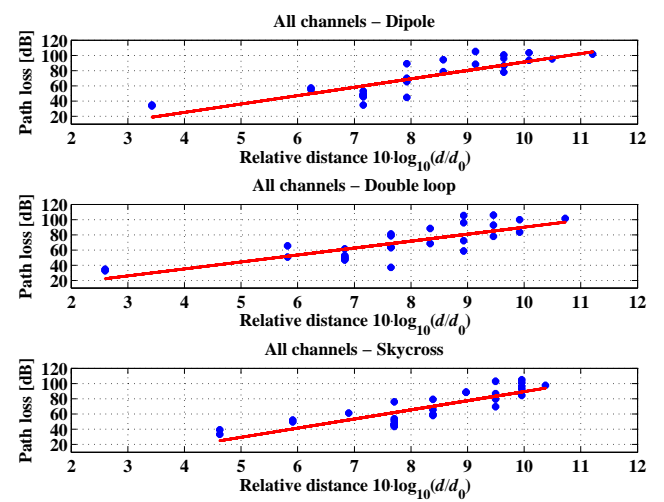

Fig. 4. Empirical and fitted PLs with the focus on all channels.
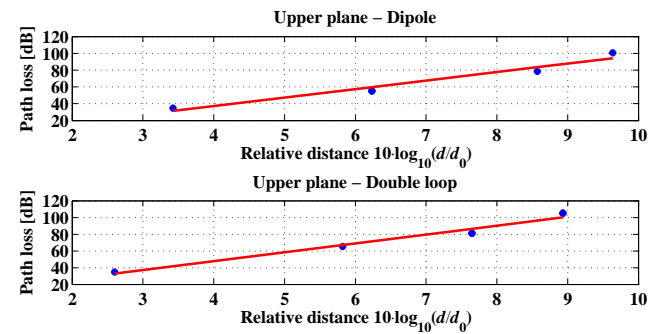

Upper plane - Skycross

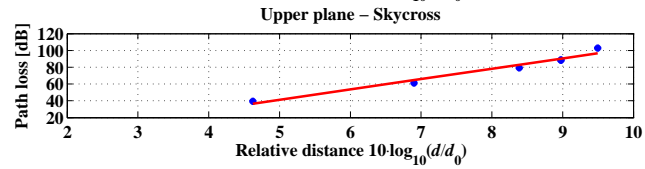

Fig. 5. Empirical and fitted PLs with the focus on the upper plane.

between 9.2-12.7. This is considerably higher value as reported in, e.g., [8, 9] with quite a similar kind of setup. However, [9] is measured in an office environment. When comparing separate empirical PLs values between [9] and Figs. 4 and 5 it can be noted that in short distances the PLs in [9] are higher than in Figs. 4 and 5 but at longer distances when the antennas are deeply in non-line-of-sight conditions, the PL values are lower in [9]. In short distances the explanation may be the antenna- body distances that was smaller in [9] $(0-10 \mathrm{~mm})$ as in our experiment. Shorter antenna body gaps affect not only on 
TABLE II. PATH LosS MOdEL PARAMETERS

\begin{tabular}{|c|c|c|c|c|}
\cline { 3 - 5 } \multicolumn{2}{c|}{} & \multicolumn{3}{c|}{ Parameters } \\
\hline Antenna & Focus & $\boldsymbol{n}$ & $\boldsymbol{P L}\left(\mathbf{d}_{\mathbf{0}}\right)$ & $\boldsymbol{\sigma}$ \\
\hline \multirow{3}{*}{ Dipole } & Upper & 10.1 & $-3.3 \mathrm{~dB}$ & $5.2 \mathrm{~dB}$ \\
\cline { 2 - 5 } & Lower & 12.7 & $-13.5 \mathrm{~dB}$ & $4.6 \mathrm{~dB}$ \\
\cline { 2 - 5 } & All & 11.0 & $-18.8 \mathrm{~dB}$ & $12.8 \mathrm{~dB}$ \\
\hline \multirow{3}{*}{ Double loop } & Upper & 10.6 & $5.2 \mathrm{~dB}$ & $4.2 \mathrm{~dB}$ \\
\cline { 2 - 5 } & Lower & 10.7 & $-1.3 \mathrm{~dB}$ & $6.4 \mathrm{~dB}$ \\
\cline { 2 - 5 } & All & 9.2 & $-1.7 \mathrm{~dB}$ & $13.3 \mathrm{~dB}$ \\
\hline \multirow{3}{*}{ Skycross } & Upper & 12.4 & $-20.7 \mathrm{~dB}$ & $4.1 \mathrm{~dB}$ \\
\cline { 2 - 5 } & Lower & 12.0 & $-20.0 \mathrm{~dB}$ & $2.5 \mathrm{~dB}$ \\
\cline { 2 - 5 } & All & 12.0 & $-30.6 \mathrm{~dB}$ & $12.0 \mathrm{~dB}$ \\
\hline
\end{tabular}

the antenna matching but also channel PL values $[12,13]$. At longer distances, the office walls may reflect signal to the receiver and may be the dominating path for signal energy to arrive to the receiver. Then the PL would saturate to a lower PL value than the first arriving path around the body in reality has. This phenomenon has been discussed also in [10].

Comparison between the antennas shows that the double loop antenna has generally speaking the lowest PL exponent. On the other hand, during data processing Skycross was the only antenna where no data needed to be discarded since the PL also in extreme links $A-5$ and B-10 did not exceed $110 \mathrm{~dB}$. The standard deviations $\sigma$ do not show clear differences between the antennas. No clear difference between the upper and lower horizontal planes can be noted although the body curvature has a slight difference between them.

The scattering term $\mathrm{S}$ is often reported to be normally distributed [10] in generic PL models. In [16], it was noted that in body area channels some other distribution may model it better. We normalized the empirical PL points with respect to the fitting line in Figs. 4 and 5 and fitted the normalized PLs with 17 distributions as was done in [16]. The fittings were ranked by using the second order Akaike information criteria $A I C_{\mathrm{c}}$ expressed as [18]

$$
A I C_{\mathrm{c}}=-2 \log \left(L\left(\theta^{\prime}\right)\right)+2 K\left(\frac{n}{n-K-1}\right),
$$

where $L\left(\theta^{\prime}\right)$ is the likelihood function of the estimated parameters $\theta^{\prime}, \log (\cdot)$ is the natural $\operatorname{logarithm}, K$ is the number of estimable parameters in a distribution and $n$ is the sample size. The distribution with the lowest $A I C_{\mathrm{c}}$ is selected as the best fit.

Fitting succeeded with the normal $(\mathrm{N})$, generalized Pareto (GP), generalized extreme value (GE), extreme value (EV), logistic (L) and t-location scale (TL) distributions. No distribution was the best in all cases as noted in Table III where the best distribution is on the left and the worst on the right in each case. GP is the best option in six cases, GE in two and normal distribution in one case.

In the all channels case with the double loop the difference in the $A I C_{\mathrm{c}}$ values is small between $\mathrm{N}$ and GE (452.06 vs. 452.27) indicating a small difference between the models. This can be verified by generating random samples to model $S$ from both $\mathrm{N}$ and GE distributions, adding them to the red fitting line in Figs. 4 and 5 and then performing the PL polynomial fitting.
TABLE III. GOODNESS OF FITS IN ASCENDING ORDER

\begin{tabular}{|c|c|c|}
\hline Antenna & Focus & Distributions \\
\hline \multirow{3}{*}{ Dipole } & Upper & GE-GP-N-EV-L-TL \\
\cline { 2 - 3 } & Lower & GP-GE-EV-L-N-TL \\
\cline { 2 - 3 } & All & GP-N-GE-TL-L-EV \\
\hline \multirow{3}{*}{ Double loop } & Upper & GP-GE-N-EV-L-TL \\
\cline { 2 - 3 } & Lower & GP-GE-EV-N-L-TL \\
\cline { 2 - 3 } & All & N-GE-GP-TL-L-EV \\
\hline \multirow{3}{*}{ skycross } & Upper & GE-GP-N-L-EV-TL \\
\cline { 2 - 3 } & Lower & GP-GE-N-L-EV-TL \\
\cline { 2 - 3 } & All & GP-GE-EV-N-TL-L \\
\hline
\end{tabular}

Fig. 6 shows the simulated data with $S$ modeled according to $\mathrm{N}$ and GE. Also the worst fit, $\mathrm{EV},\left(A I C_{\mathrm{c}}=455.17\right)$ is shown. Fifty random variables are generated at each distance. The resulting $n$ and $P L\left(d_{0}\right)$ are shown in the titles of Fig. 6. The difference between $\mathrm{N}$ and GE is small. Even the poorest fit with EV does not differ much from the two best ones. Therefore GE is selected for the Skycross/all channels case to keep the model number as two in all focus cases. The selected distributions are bolded in Table III.

The probability density function (PDF) of GP is [19]

$$
\begin{aligned}
& f(x \mid k, \alpha, \beta)= \\
& \left\{\begin{array}{l}
\left(\frac{1}{\alpha}\right)\left(1+k \frac{(x-\beta)}{\alpha}\right)^{-1-\frac{1}{k}}, \text { if } k>0, x>\beta \\
\text {, if } k<0, \beta<x<-\frac{\alpha}{k},
\end{array}\right. \\
& \left(\frac{1}{\alpha}\right) \mathrm{e}^{-\frac{(x-\beta)}{\alpha}} \text {,if } k=0
\end{aligned}
$$

where $k$ is the shape, $\alpha$ is the scale and $\beta$ is the threshold parameter.

GE has the PDF [19]

$$
\begin{aligned}
& f(x \mid m, \varphi, \lambda)= \\
& \left(\frac{1}{\lambda}\right) \exp \left[-\left(1+m \frac{(x-\varphi)}{\lambda}\right)^{-\frac{1}{m}}\right]\left(1+m \frac{(x-\varphi)}{\lambda}\right)^{-1-\frac{1}{m}}
\end{aligned}
$$

for

$$
1+m \frac{(x-\varphi)}{\lambda}>0 \quad \text { and } \quad m \neq 0 \text {, }
$$
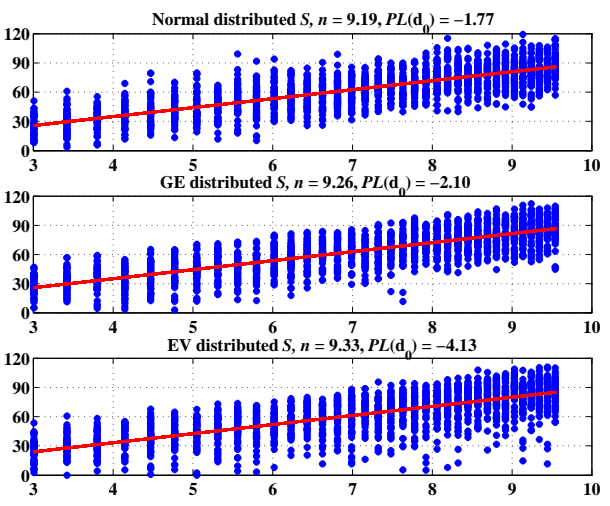

Fig. 6. Normal, GE and EV modeled $S$ and PL model for the double loop. 
TABLE IV. DISTRIBUTION PARAMETERS

\begin{tabular}{|c|c|c|c|c|}
\hline Antenna & Focus & \multicolumn{3}{|c|}{ Parameters } \\
\hline \multirow{3}{*}{ Dipole } & Upper & $m=-1.1527$ & $\varphi=5.7667$ & $\lambda=1.2041$ \\
\cline { 2 - 5 } & Lower & $k=-1.2262$ & $\alpha=14.7216$ & $\beta=-8.2410$ \\
\cline { 2 - 5 } & All & $k=-1.0891$ & $\alpha=52.6885$ & $\beta=-24.9175$ \\
\hline \multirow{3}{*}{ Double loop } & Upper & $k=-1.4808$ & $\alpha=15.6169$ & $\beta=-5.3106$ \\
\cline { 2 - 5 } & Lower & $m=-1.2846$ & $\varphi=8.9081$ & $\lambda=-0.9608$ \\
\cline { 2 - 5 } Skycross & All & $m=-0.3903$ & $\varphi=13.7318$ & $\lambda=-3.9650$ \\
\hline & Upper & $m=3.5018$ & $\varphi=0.3112$ & $\lambda=-3.6618$ \\
\cline { 2 - 5 } & Lower & $k=-1.2170$ & $\alpha=7.8080$ & $\beta=-3.0703$ \\
\cline { 2 - 5 } & All & $k=-1.0147$ & $\alpha=38.2936$ & $\beta=-18.1156$ \\
\hline
\end{tabular}

where $m$ is the location, $\varphi$ the scale and $\lambda$ are the shape parameter. If $m=0$, the PDF is expressed as [19]

$$
f(x \mid m, \varphi, \lambda)=\left(\frac{1}{\lambda}\right) \exp \left[-\exp \left(-\frac{(x-\varphi)}{\lambda}\right)-\frac{(x-\varphi)}{\lambda}\right] \text {. }
$$

The distribution parameters are collected in Table IV.

\section{CONCLUSIONS AND FUTURE WORK}

The UWB signal propagation as creeping waves around a human torso is studied. The results are based on anechoic chamber measurements at the $2-8 \mathrm{GHz}$ band. Three antenna types are used: prototype dipole and double loop antennas and the commercial Skycross antenna. The antennas are attached at two horizontal planes at the chest and waist at six locations each, in total 12 locations. PL models are developed for both planes separately, and for the case when all links with the cross links between the two planes are included. The PL exponents vary between 9.2-12.7 with double loop having in overall the lowest exponent and Skycross the highest. The scattering term of the PL can be modelled with the generalized extreme value and generalized Pareto distributions.

The results indicate that the PL models for the on-body communications still need investigation. The fact that the PL exponents deviate from the previously reported values show that further measurements are needed in different environments and with different antenna types. As is known, antennas with vertical polarization with respect to the body surface produce clearly lower PL than antennas with horizontal polarization [6] as is the case in theory with the antennas used in this paper.

\section{ACKNOWLEDGMENT}

The authors would like to thank Dr. Kamya Yekeh Yazdandoost and Dr. Tommi Tuovinen for the prototype antennas design.

\section{REFERENCES}

[1] I. Oppermann, M. Hämäläinen, J. Iinatti (eds.), UWB Theory and Applications. West Sussex, England, John Wiley \& Sons, 2004, pp. 1-7.

[2] M Hämäläinen, A Taparugssanagorn, R Tesi, and J Iinatti, "Wireless medical communications using UWB," in Proc. 2009 IEEE Int. Conf. on
Ultra-Wideband (ICUWB'09), Sept. 2009, Vancouver, Canada, pp. 485 489.

[3] IEEE standard for local and metropolitan area networks, IEEE 802.15.62012 - Part 15.6: wireless body area networks, 2012.

[4] R. Paknys, and N. Wang, "Creeping wave propagation constants and modal impedance for a dielectric coated cylinder," IEEE Trans. Antennas Propag., vol. AP-34, no. 5, 1986, pp. 674-680.

[5] T. Zasowski, G. Meyer, F. Althaus, and A. Wittneben, "UWB signal propagation at the human head," IEEE Trans. Microw. Theory Techniques, vol. 54, no. 4, 2006, pp. 1836-1845.

[6] T. Alves, B. Poussot, and J.-M. Laheurte, "Analytical propagation modeling of BAN channels based on creeping wave theory," IEEE Trans. Antennas Propag., vol. 59, no. 4, 2011, pp. 1269-1274.

[7] H. Yamamoto, and T. Kobayashi, "Ultra-wideband propagation loss around a human body in various surrounding environments," in UltraWideband, Short Pulse Electromagnetics 9, F. Sabath, D.V. Giri, F. Rachidi, and A. Kaelin (Eds.), New York, Springer, 2010, pp. 11-18.

[8] A. Fort, C. Desset, J. Ryckaert, P. De Doncker, L. Van Biesen, and S. Donnay, "Ultra wide-band body area channel model," in Proc. IEEE Int. Conf. on Commun. (ICC), 2005, Seoul, Korea, pp. 2840-2844.

[9] A. Fort, J. Ryckaert, C. Desset, P. De Doncker, P. Wambacq, and L. Van Biesen, "Ultra-wideband channel model for communication around the human body," IEEE J. Sel. Areas Commun., vol. 24, no. 4, 2006, pp. 927-933.

[10] A. Fort, F. Keshmiri, G. Roqueta Crusats, C. Craeye, and C. Oestges “A body area propagation model derived from fundamental principles: analytical analysis and comparison with measurements," IEEE Trans. Antennas Propag., vol. 58, no. 2, 2010, pp. 503-514.

[11] T. Kumpuniemi, T. Tuovinen, M. Hämäläinen, K. Yekeh Yazdandoost, R. Vuohtoniemi, and J. Iinatti, "Measurement-based on-body path loss modelling for UWB WBAN communications," in Proc. $7^{\text {th }}$ Int. Symp. Medical Inform. and Commun. Technology (ISMICT), March 2013, Tokyo, Japan, pp. 233-237.

[12] T. Tuovinen, T. Kumpuniemi, K. Yekeh Yazdandoost, M. Hämäläinen, and J. Iinatti, "Effect of the antenna-human body distance on the antenna matching in UWB WBAN applications," in Proc. $7^{\text {th }}$ Int. Symp. on Medical Inform. and Commun.Technology (ISMICT), March 2013, Tokyo, Japan, pp. 193-197.

[13] T. Tuovinen, T. Kumpuniemi, M. Hämäläinen, K. Yekeh Yazdandoost, and J. Iinatti, "Effect of the antenna-body distance on the on-ext and onon channel link path gain in UWB WBAN applications," in Proc. 35th Annu. Int. Conf. IEEE Eng. in Medicine and Biology Society (EMBC), July 2013, Osaka, Japan, pp. 1242-1245.

[14] T. Kumpuniemi, M. Hämäläinen, K. Yekeh Yazdandoost, and J. Iinatti, "Measurements for body-to-body UWB WBAN radio channels," in Proc. The 9th European Conference on Antennas and Propagation (EUCAP' 2015), Apr. 2015, Lisbon, Portugal, pp. 1-5.

[15] T. Kumpuniemi, M. Hämäläinen, K. Yekeh Yazdandoost, and J. Iinatti, "Human body size and shape effect on UWB on-body WBAN radio channels - preliminary results", in: Proc. The Third Ultra Wideband for Body Area Networking Workshop (UWBAN-2014), Co-located with the 9th Int.Conf. on Body Area Networks (BodyNets-2014), Sept.-Oct. 2014, London, United Kingdom, pp. 1-7.

[16] T. Kumpuniemi, M. Hämäläinen, K. Yekeh Yazdandoost, and J. Iinatti, "Categorized UWB on-body radio channel modeling for WBANs". Progress in Electromagnetic Research B, Vol. 67, 2016, pp. 1-16.

[17] A. F. Molisch et al., "IEEE 802.15.4a channel model-final report," IEEEP802.15 Working Group for Wireless Personal Area Networks (WPANs) (IEEE 802.15-04-0662-01-04a, Sept. 2004).

[18] K. P. Burnham, and D. R. Anderson, Model Selection and Multimodel Inference, A Practical Information-Theoretic Approach, 2nd ed., New York, USA, Springer-Verlag, 2002, pp. 60-72.

[19] Mathworks, MATLAB, Documentation Center, Statistics and Machine Learning Toolbox, (November 2016). [Online] available: http://se.mathworks.com/help/stats/index.html. 